\title{
Impairment of DYRK2 by DNMT1-mediated transcription augments carcinogenesis in human colorectal cancer
}

\author{
TOMOTAKA KUMAMOTO $^{1,2}$, KOHJI YAMADA ${ }^{1}$, SAISHU YOSHIDA $^{1}$, KATSUHIKO AOKI $^{1}$, \\ SHINICHI HIROOKA ${ }^{3}$, KEN ETO ${ }^{2}$, KATSUHIKO YANAGA ${ }^{2}$ and KIYOTSUGU YOSHIDA ${ }^{1}$
}

Departments of ${ }^{1}$ Biochemistry, ${ }^{2}$ Surgery and ${ }^{3}$ Pathology, The Jikei University School of Medicine, Tokyo 105-8461, Japan

Received December 4, 2019; Accepted February 21, 2020

DOI: $10.3892 /$ ijo.2020.5020

\begin{abstract}
Dual specificity tyrosine-phosphorylation-regulated kinase 2 (DYRK2) is a protein kinase that functions as a novel tumor suppressor. Previous studies have reported that DYRK2 expression is decreased in colorectal cancer compared with adjacent non-tumor tissues. However, the regulatory mechanisms by which the expression of DYRK 2 is diminished remain unknown. The aim of the present study was to determine the regulatory mechanisms of DYRK2 expression. The present study identified the promoter regions of the DYRK2 gene and demonstrated that they contained $\mathrm{CpG}$ islands in human cancer cells. In addition, the DYRK2 promoter region exhibited a higher level of methylation in colorectal cancer tissues compared with healthy tissues from clinical samples. DYRK2 expression was increased at the mRNA and protein level in colorectal cancer cell lines by treatment with 5-Azacytidine, a demethylating agent. The results further demonstrated that knockdown of DNA methyltransferase (DNMT) 1 elevated DYRK2 expression in colorectal cancer cell lines. A colitis-related mouse carcinogenesis model also exhibited a lower DYRK2 level in colorectal cancer tissues compared with adjacent non-tumor tissues. In this model, nuclear staining of DNMT1 was detected in colorectal cancer cells, whereas a cytoplastic distribution pattern of DNMT1 staining was exhibited in healthy tissue. Overall, these findings suggested that DYRK2 expression was downregulated
\end{abstract}

Correspondence to: Professor Kiyotsugu Yoshida, Department of Biochemistry, The Jikei University School of Medicine, 3-25-8 Nishi-Shinbashi, Minato, Tokyo 105-8461, Japan

E-mail: kyoshida@jikei.ac.jp

Abbreviations: DYRK2, dual specificity tyrosine-phosphorylationregulated kinase 2; DNMT, DNA methyltransferase; CRC, Colorectal cancer; TSS, transcription start site; ChIP, chromatin immunoprecipitation; Aza, 5-Azacytidine; AOM, azoxymethane; DSS, dextran sodium sulfate

Key words: DYRK2, colorectal cancer, DNMT1, DNA methylation, 5-Azacytidine via transcriptional regulation by DNMT1 to elevate the proliferation of colorectal cancer cells.

\section{Introduction}

Colorectal cancer (CRC) is the third most common malignancy worldwide, accounting for $\sim 1.8$ million new cases and $\sim 880,000$ deaths in 2018 (1). The carcinoma progression sequence is universally accepted and originates from benign polyps or dysplastic lesions, which develop into advanced adenoma and invasive carcinoma (2). In the past four decades, the mortality rate of CRC has decreased, possibly due to improved cancer screening programs, surgical techniques and therapeutic strategies for early- and advanced-stage disease (3). However, patients with an advanced stage have a poor prognosis, with the 5-year survival rate of $12.5 \%(4,5)$.

Tumor progression and distant metastasis are the primary causes of death in patients with CRC and are complicated by various genetic and epigenetic changes (6,7). Epigenetic modification is tightly associated with molecular changes in DNA that do not occur from alterations in the DNA proper $(8,9)$. Epigenetic alterations coordinately occur with other genetic changes, such as chromosomal rearrangements or genomic mutations, and affect the transcriptomics, genomics and proteomics that drive cancer phenotypes (10). Such epigenetic alterations involve a range of mechanisms, including DNA methylation, histone modification, microRNAs, chromatin remodeling and other chromatin components (11). Epigenetics is essential for research into processes underlying various diseases, including cancer (12).

DNA methylation is the most researched epigenetic mechanism (8), during which a methyl group is added to DNA nucleotides by DNA methyltransferase (DNMT). DNMT1 is the largest DNMT that participates in methylation (13). DNMT3 functions as a de novo methyltransferase and comprises two related proteins encoded by the DNMT3A and DNMT3B genes (14). DNA methylation is a stable modification conserved through cellular divisions by DNMTs (13).

In humans, DNA methylation generally occurs at the five-carbon position of the cytosine pyrimidine ring preceding guanine, termed dinucleotide $\mathrm{CpG}$ (8). Hypo- and hypermethylation of the $\mathrm{CpG}$ sites regulate transcriptional events and alter specific gene or DNA segment expression (15). Consequently, DNA methylation of tumor suppressor genes causes progression of several types of carcinoma (15). 
Dual specificity tyrosine-phosphorylation-regulated kinase 2 (DYRK2), a protein kinase of the DYRK family, is mainly localized in the cytoplasm and exhibits serine/threonine kinase activity in cells (16). DYRK2 serves as a proapoptotic kinase through $\mathrm{p} 53$ phosphorylation at Ser46 for inducing apoptotic cell death as a response to acute DNA damage $(17,18)$. In addition to its role in apoptosis, DYRK2 regulates cell cycle progression by the degradation of c-Myc, c-Jun, telomere reverse transcriptase and katanin p60 (19-22). As cell cycle inhibition induces tumorigenesis, DYRK2 may serve a role in tumor suppression (16). Clinically, previous studies have reported decreased DYRK2 expression in advanced breast (22) and lung cancer (23), hepatocellular carcinoma (24), colorectal cancer (25) and metastatic liver lesions of colorectal cancer (26). Accordingly, the regulation of DYRK2 expression may be necessary for cell proliferation. Nevertheless, the molecular mechanisms underlying DYRK2 expression remain largely unknown.

The present study aimed to identify the DYRK2 promoter regions and determine whether the promoter region was subjected to DNA methylation in CRC cell lines and patient tissues. In addition, the present study aimed to clarify whether DYRK2 expression was upregulated by the inhibition of DYRK2 methylation at the promoter region, resulting in inhibition of tumor cell proliferation in CRC.

\section{Materials and methods}

Patient samples. The study protocol was approved by the Jikei University School of Medicine Ethics Review Committee (approval no. 24-315 7081; Tokyo, Japan). All patients provided written informed consent that their colorectal samples would be utilized for investigative purposes. CRC and adjacent non-cancerous tissues were obtained from resected specimens of a total of 80 patients at the Jikei University Hospital between October 2014 and November 2015. The distance between cancer and adjacent non-cancerous tissue was $\leq 5 \mathrm{~cm}$. The inclusion criteria were patients who were diagnosis with CRC by preoperative diagnosis and who underwent tumor resection for therapeutic purposes. The exclusion criteria were patients $<18$ years and unable to provide informed consent. The mean age of patients was 68.6 years (range, 39-90 years). For mRNA extraction, samples were frozen immediately after surgical resection and stored at $-80^{\circ} \mathrm{C}$ until use. The clinicopathological information of the patients included in the present study is presented in Table SI.

Cell culture and treatment. HCT116 cell lines (human CRC cells) were purchased from ATCC. The cells were cultured in Dulbecco's modified Eagle's medium (DMEM; Nacalai Tesque, Inc.) supplemented with 10\% FBS (Biological Industries) and $1 \%$ penicillin and streptomycin at $37^{\circ} \mathrm{C}$ in a $5 \% \mathrm{CO}_{2}$ incubator. DLD-1, LS180, SW480 and SW620 cells were obtained from ATCC and cultured in RPMI-1640 medium (Nacalai Tesque, Inc.) with $10 \% \mathrm{FBS}, 1 \%$ penicillin and streptomycin at $37^{\circ} \mathrm{C}$ in a $5 \% \mathrm{CO}_{2}$ incubator.

5-Azacytidine (Aza) treatment. Aza was purchased from Wako Pure Chemical Industries (cat. no. 016-25361) and dissolved in DMSO for stock and working solution preparation. Human
CRC cell lines (HCT116, DLD-1, LS180, SW620 and SW480) were treated with Aza at concentrations ranging between 1 and $20 \mu \mathrm{M}$ for 1 or 2 days. The control cells were treated with DMSO as a vehicle control for the same time.

Cell transfection. DYRK2 small interfering (si)RNA (\#1, Qiagen GmbH; \#2, Invitrogen; Thermo Fisher Scientific, Inc.), negative control siRNA (Invitrogen; Thermo Fisher Scientific, Inc.) and DNMT1 siRNA (Sigma-Aldrich; Merck $\mathrm{KGaA}$ ) were used to establish cell lines with a transient knockdown. The siRNA sequences are stated in Table SII. To identify cell lines with transient DYRK2 overexpression, a pCDNA3 flag vector was used; a pCDNA3 flag empty vector was used as the negative control. The HCT116 cells were plated and cultured to $60-80 \%$ confluency prior to transfection. The vectors $(500 \mathrm{nM})$ and siRNAs $(5 \mathrm{nM})$ were transfected to HCT116 cells using the Lipofectamine ${ }^{\circledR}$ RNAiMAX Reagent (Invitrogen; Thermo Fisher Scientific, Inc.) according to the manufacturer's instructions. The expression of each protein was determined $48 \mathrm{~h}$ post-transfection by western blotting.

Western blotting. Western blotting using all the cell lines was conducted as previously described (19). The Bradford protein assay was used to measure the concentration of total protein in samples. The concentration of protein was $1 \mathrm{mg} / \mathrm{ml}$. The amount of protein loaded was 20-30 $\mu \mathrm{g}$ per lane. The protein samples were separated by SDS-PAGE on $7.5 \%$ gels and transferred to nitrocellulose membranes. Blocking one (cat. no. 03953-66; Nacalai Tesque, Inc.) was used for the blocking of the membranes for $1 \mathrm{~h}$ at room temperature. The primary antibodies used for immunoblotting were polyclonal rabbit anti-DYRK2 (1:1,000; cat. no. HPA027230; Sigma-Aldrich; Merck KGaA), monoclonal mouse anti-DNMT1 (1:1,000; clone H-12; cat. no. sc-271729; Santa Cruz Biotechnology, Inc.), monoclonal mouse anti-tubulin (1:1,500; clone B-5-1-2; cat. no. T5168; Sigma-Aldrich; Merck KGaA) and monoclonal mouse anti-GAPDH (1:1,500; clone 6C5; cat. no. MAB374; Sigma-Aldrich; Merck KGaA). The membranes were incubated with the primary antibodies overnight at $4^{\circ} \mathrm{C}$ and washed three times for 15 min with TBS $+0.05 \%$ Tween-20. The secondary antibodies were monoclonal horseradish peroxidase-conjugated goat anti-rabbit (1:1,000; clone eB182; cat. no. 18-8816-31; eBioscience) and goat anti-mouse (1:1,000; clone eB144; cat. no. 18-8817-31; eBioscience;). The membranes were incubated for $1 \mathrm{~h}$ at room temperature. All western blotting experiments were conducted at least three times.

$R N A$ extraction and reverse transcription-quantitative $P C R$ $(R T-q P C R)$. Total RNA from human and mouse tissues was extracted using the RNeasy Plus Universal Mini Kit (Qiagen $\mathrm{GmbH}$ ) according to the manufacturer's protocol. RNA from all cell lines was extracted using the TRIsure reagent (Nippon Gene Co., Ltd.). Total RNA was reverse transcribed into cDNA using the PrimeScript 1st Strand cDNA Synthesis kit (Takara Bio, Inc.) and each primer pair $(50 \mu \mathrm{M})$. The primer sequences are summarized in Table SII. The reaction conditions were $65^{\circ} \mathrm{C}$ for $5 \mathrm{~min}, 4^{\circ} \mathrm{C}$ for $5 \mathrm{~min}, 42^{\circ} \mathrm{C}$ for $60 \mathrm{~min}$ and $95^{\circ} \mathrm{C}$ for 5 min. SYBR ${ }^{\circledR}$ Green PCR Master Mix (Applied Biosystems; Thermo Fisher Scientific, Inc.) was used for qPCR according to the manufacturer's instructions. Amplification and detection 
were performed using a PikoReal 96 Real-Time PCR System (Thermo Fisher Scientific, Inc.). The thermocycling conditions were as follows: $95^{\circ} \mathrm{C}$ for $10 \mathrm{~min}$, followed by 40 cycles of $95^{\circ} \mathrm{C}$ for $15 \mathrm{sec}, 60^{\circ} \mathrm{C}$ for $30 \mathrm{sec}$ and $72^{\circ} \mathrm{C}$ for $30 \mathrm{sec}$. GAPDH was used as an internal control. The mRNA expression level was calculated using the $2^{-\Delta \Delta \mathrm{Cq}}$ method (27).

Generation of DYRK2 promoter constructs and luciferase reporter assay. The transcription start site (TSS) of DYRK2 was identified using the DataBase of Transcriptional Start Sites (https://dbtss.hgc.jp). To produce the luciferase reporter gene construct containing the DYRK2 promoter, genomic DNA was purified from the HCT116 human CRC cell line using a DNeasy Blood \& Tissue Kit (Qiagen GmbH). Several DNA fragments around the DYRK2 promoter were obtained from the KAPA HiFi HotStart ReadyMix (Kapa Biosystems; Roche Diagnostics) using genomic DNA as a template and primer sets of the forward primer with an incorporated KpnI site or the reverse primer with an incorporated NheI site. The primer sequences are summarized in Table SII. The reaction conditions were as follows: $95^{\circ} \mathrm{C}$ for $5 \mathrm{~min}$, followed by 30 cycles of $98^{\circ} \mathrm{C}$ for $20 \mathrm{sec}, 65^{\circ} \mathrm{C}$ for $15 \mathrm{sec}$ and $72^{\circ} \mathrm{C}$ for $3 \mathrm{~min}$, and a final extension at $72^{\circ} \mathrm{C}$ for $5 \mathrm{~min}$. The resultant constructs were amplified by PCR using the BigDye v3.1 Terminator Cycle Sequencing kit (Applied Biosystems; Thermo Fisher Scientific, Inc.). The reaction conditions were as follows: $96^{\circ} \mathrm{C}$ for $30 \mathrm{sec}$, followed by 25 cycles of $96^{\circ} \mathrm{C}$ for $10 \mathrm{sec}, 50^{\circ} \mathrm{C}$ for $5 \mathrm{sec}$ and $60^{\circ} \mathrm{C}$ for $4 \mathrm{~min}$. The constructs were confirmed by Sanger sequencing with a 3730xl DNA Analyzer (Applied Biosystems; Thermo Fisher Scientific, Inc.). The PCR product was ligated into the pGL3-Basic luciferase vector (Promega Corporation).

For the luciferase reporter assay, HCT116 cells were seeded in triplicate into a 96 -well plate $\left(5 \times 10^{3}\right.$ cells/well $)$ and transfected with pGL3 plasmids $48 \mathrm{~h}$ later. The pGL3 plasmids contained each construct fragment, along with a Renilla luciferase plasmid (pRL-TK; Promega Corporation) as the normalization control using the FuGENE HD (Promega Corporation) transfection reagent. A pGL3-Basic vector without an insert was used as a negative control. At $24 \mathrm{~h}$ post-transfection, the luciferase activity was determined using the Dual-Luciferase Reporter Assay System (Infinite 200PRO; Tecan Group, Ltd.). Luciferase activity was calculated and expressed as relative light units. The $\mathrm{CpG}$ islands were confirmed by MethPrimer (http://www.urogene. org/methprimer2/), which is used to design PCR primers for methylation mapping.

Chromatin immunoprecipitation (ChIP) assay. The ChIP assay was performed using a SimpleChIP ${ }^{\circledR}$ Enzymatic Chromatin IP kit (cat. no. 9003; Cell Signaling Technology, Inc.) according to the manufacturer's instructions. HCT116 cells were fixed with $37 \%$ formaldehyde for $10 \mathrm{~min}$ at room temperature, washed with $20 \mathrm{ml}$ ice-cold $1 \mathrm{X}$ PBS. The lysate was treated with Micrococcal Nuclease (Takara Bio, Inc.) and sonicated (20 sec on, 1 min off for 3 times) to obtain fragmented DNA for subsequent PCR. The DNA was subjected to PCR with specific primers of the DYRK2 promoter region. The primer sequences are stated in Table SII. PCR was performed using the following conditions: $95^{\circ} \mathrm{C}$ for $15 \mathrm{~min}$, followed by 40 cycles of $94^{\circ} \mathrm{C}$ for $30 \mathrm{sec}, 54^{\circ} \mathrm{C}$ for $30 \mathrm{sec}$ and $72^{\circ} \mathrm{C}$ for $30 \mathrm{sec}$, and a final step of $72^{\circ} \mathrm{C}$ for $10 \mathrm{~min}$.

MTS assay. HCT116 cells were seeded into 96-well plates ( $1 \times 10^{4}$ cells/well), and MTS assay was conducted using a CellTiter 96 AQ Solution Cell Proliferation Assay Kit (Promega) according to the manufacturer's protocol. The absorbance was measured at $490 \mathrm{~nm}$ with a multiple counter (Infinite 200PRO; TECAN).

DNA methylation analysis. Genomic DNA was purified from the HCT116 cells or human tissues using a DNeasy Blood \& Tissue kit (Qiagen $\mathrm{GmbH}$ ). The DNA was sonicated (30 sec on, 1 min off for 10 times) to obtain fragmented DNA. The methylated and unmethylated DNA fragments in whole DNA samples were enriched by an EpiXplore Methylated DNA Enrichment Kit (Takara Bio, Inc.) according to the manufacturer's instructions. The fragmented DNA was treated with SYBR ${ }^{\circledR}$ Green PCR Master Mix (Applied Biosystems; Thermo Fisher Scientific, Inc.) and a DYRK2 primer set for qPCR. The primers sequences are summarized in Table SII. The products were analyzed with a PikoReal 96 Real-Time PCR System. The thermocycling conditions were as follows $95^{\circ} \mathrm{C}$ for $10 \mathrm{~min}$, followed by 40 cycles of $95^{\circ} \mathrm{C}$ for $15 \mathrm{sec}, 60^{\circ} \mathrm{C}$ for $30 \mathrm{sec}$ and $72^{\circ} \mathrm{C}$ for $30 \mathrm{sec}$. The products were calculated by the percentage of input using $\mathrm{Ct}$ values as follows: Percent input $=2 \% \times 2^{\text {(Ct 2\% input sample-Ct IP sample) }}$. The input sample used was $2 \%$.

Animals and treatment. The animal experiment was preformed following the Guidelines for the Proper Conduct of Animal Experiments of the Science Council of Japan and approved by the Institutional Animal Care and Use Committee of Jikei University (approval no. 2018-015). Female 5-week-old C57/BL6 mice (Charles River Laboratories Japan) were habituated to the new environment for 7 days after arrival. Azoxymethane (AOM) was obtained from Sigma-Aldrich; Merck KGaA, and dextran sodium sulfate (DSS) was purchased from MP Biomedicals, LLC. A total of 15 mice were divided into 3 groups. The mice were administered a single intraperitoneal injection of AOM (10 mg/kg body weight) at the age of 6 weeks. One week after the AOM injection, the mice started to receive $2 \%$ DSS in their drinking water for 7 days. The mice were sacrificed by isoflurane overdose at 10 , 15 , or 20 weeks after the AOM injection treatment. Tumor and adjacent non-cancerous tissues were collected from the colon and rectum of the mice.

Hematoxylin and eosin (HE) staining and immunohistochemistry. The colorectal tissues of mice were fixed with $4 \%$ paraformaldehyde in $20 \mathrm{mM}$ HEPES buffer ( $\mathrm{pH} 7.5$ ) overnight at $4^{\circ} \mathrm{C}$, followed by immersion in $30 \%$ trehalose in $20 \mathrm{mM}$ HEPES to cryoprotect the tissue. The samples were embedded in Tissue-Tek O.C.T. Compound (Sakura Finetek Japan Co., Ltd.) and frozen in dry ice-acetone. For HE staining, frozen $6-\mu \mathrm{m}$ sections were washed with water, followed by staining with hematoxylin and eosin for $7 \mathrm{~min}$ and $10 \mathrm{sec}$ at room temperature, respectively. After dehydration, the sections were sealed for microscopy observation. For immunohistochemistry, frozen $6-\mu \mathrm{m}$ sections were treated with ImmunoSaver 
(Nisshin EM Co., Ltd.) for $1 \mathrm{~h}$ at $80^{\circ} \mathrm{C}$ for antigen retrieval and washed with HEPES buffer [20 mM HEPES (pH 7.5), $100 \mathrm{mM} \mathrm{NaCl}$. Blocking was performed by incubating with $0.4 \%$ Triton X-100 and 10\% FBS in HEPES buffer for 60 min at room temperature. The sections were incubated with rabbit anti-human DYRK2 (dilution 1:400; cat. no. HPA027230; Sigma-Aldrich; Merck KGaA) and mouse anti-mouse DNMT1 antibody (dilution 1:300; clone H-12; cat. no. sc-271729; Santa Cruz Biotechnology, Inc.) overnight at $4^{\circ} \mathrm{C}$. Following washing with HEPES buffer, the sections were incubated with $\mathrm{Cy}^{\circledR} 3$-conjugated donkey anti-rabbit (dilution 1:500; cat. no. 711-165-152; Jackson ImmunoResearch Laboratories, Inc.) and $\mathrm{Cy}^{\circledR} 5$-conjugated donkey anti-mouse $\operatorname{IgG}$ (dilution 1:500; cat. no. 715-175-151; Jackson ImmunoResearch Laboratories, Inc.) for $2 \mathrm{~h}$ at $4^{\circ} \mathrm{C}$. The sections were washed and mounted with VECTASHIELD ${ }^{\circledR}$ Mounting Medium (Vector Laboratories, Inc.) with 4,6'-diamidino-2-phenylindole dihydrochloride (DAPI). Immunofluorescence was observed under a BZ-X800 fluorescence microscope (x20 magnification; $n=8$ fields per sample; Keyence Corporation).

Statistical analysis. The data are presented as the mean \pm standard deviation. SPSS 25.0 statistical software (IBM Corp) was used for statistical analyses. One-way ANOVA with a Tukey's multiple comparisons test and two-tailed Student's t-test were used to identify the difference between two groups in the results of the luciferase reporter gene assay, RT-qPCR, and the MTS assay. Paired Student's t-test was used to compare tumor and adjacent normal tissues; unpaired Student's t-test was used for cell group analysis. Associations between DYRK2 expression levels and clinicopathological parameters were analyzed using the Pearson's $\chi^{2}$ test. $\mathrm{P}<0.05$ was considered to indicate a statistically significant difference.

\section{Results}

DYRK2 expression is decreased in human CRC tissues. To investigate the roles of DYRK2 in tumorigenesis, DYRK2 expression was evaluated in several matched pairs of tumor and adjacent non-cancerous tissues from patients who underwent surgery for CRC. Western blotting analysis revealed that DYRK2 expression was downregulated in CRC tissues compared with that in adjacent non-cancerous tissues (Fig. 1A).

RT-qPCR was performed using cancer tissue and adjacent non-cancerous tissue samples from 80 patients with CRC. The DYRK2 mRNA level was significantly lower in cancer tissues compared with that in adjacent non-cancerous tissues (Fig. 1B). These results suggested a negative association between DYRK2 expression and tumor development or progression in CRC. In addition, clinicopathological analysis was performed with $80 \mathrm{CRC}$ samples (Table SI). Tumors were staged according to the 8th edition of the American Joint Committee on Cancer staging system (28). The clinical characteristics of CRC included 14 stage I (17.5\%), 25 stage II (31.2\%), 32 stage III (40\%) and 9 stage IV (11.3\%) cases of CRC, and 35 of the 80 patients with CRC (43.7\%) exhibited high DYRK2 expression ( $>0.5$ of the mRNA level of adjacent normal tissue) and 45 patients (56.3\%) exhibited low DYRK2 expression.
The samples were divided into two groups based on DYRK2 expression levels, and the clinicopathological characteristics of the patients in the high- $(>0.5$ of the mRNA level of adjacent normal tissue) and low- ( $\leq 0.5$ of the mRNA level of adjacent normal tissue) expression groups were analyzed by the $\chi^{2}$-test (Table I). A strong association was observed between DYRK2 expression and the clinicopathological features of patients with $\mathrm{CRC}$, including age $(\mathrm{P}=0.003)$ and tumor location $(\mathrm{P}=0.030)$ (Table I). By contrast, no associations were observed between DYRK2 mRNA expression and sex, clinical stage, Tumor-Node-Metastasis classification or pathological differentiation (Table I) (28).

DYRK2 promoter regions are methylated in CRC. To identify the molecular mechanism of DYRK2 transcriptional regulation, the basal transcriptional regulation of the DYRK2 promoter region was analyzed from 2,000 bp upstream to $200 \mathrm{bp}$ downstream of the TSS. To achieve this, a DNA fragment between $-2,000$ and +200 bp around the TSS was cloned into the pGL3 vector containing firefly luciferase (Fig. 2A). In addition, various truncated promoter constructs were generated, as presented in Fig. 2B. Luciferase activity was high in the P5, P6 and P7 fragments in HCT116 cells. However, further truncation (P1-P4) significantly reduced the reporter activity compared with that observed with P5 (Fig. 2B). These findings suggested that one of the promoters for DYRK2 was located in the region between at least-150 and -50 .

A ChIP assay was performed using an RNA polymerase II antibody to examine whether the $-150 /+1$ region exhibited promoter functions. The results demonstrated that RNA polymerase II was bound to the region (Fig. 2C), further suggesting that the $-150 /+1$ region was one of the promoter regions for the DYRK2 gene.

Bioinformatics analysis was next performed using the DYRK2 promoter region in HCT116 cells. A large proportion of cytosine and guanine was detected in the $-150 /+1$ region (Fig. 2D). These results indicated that the DYRK2 promoter region contained a $\mathrm{CpG}$ island enriched in the $\mathrm{CpG}$ repeats (Fig. 2D). This result suggested that DNA methylation may occur within the DYRK2 promoter.

DNA methyltransferase inhibitor induces DYRK2 expression and suppresses proliferation in CRC cell lines. To determine whether DNA methylation was involved in the DYRK2 expression, the effects of DNA methyltransferase inhibitors on DYRK2 mRNA expression were investigated. RT-qPCR analysis revealed that treatment with Aza upregulated the transcription of DYRK2 in a dose-dependent manner (Fig. 3A). Similar results for the effect of Aza treatment were obtained in five CRC cell lines HCT116, DLD-1, LS180, SW480 and SW620. DYRK2 mRNA expression was significantly increased following treatment with $5 \mu \mathrm{M}$ Aza in all cell lines (Fig. 3B). In addition, when the HCT116 cells were treated with $10 \mu \mathrm{M}$ Aza for $48 \mathrm{~h}$, the DYRK2 protein level was upregulated (Fig. 3C). The DYRK2 promoter area of the HCT116 cells treated with $10 \mu \mathrm{M}$ Aza exhibited a lower level of methylation compared with that in cells treated with the vehicle (Fig. 3D).

To assess whether cell proliferation was associated with DYRK2 expression in the CRC cell lines, HCT116 cells were transfected with DYRK2 siRNA or control 
Table I. Clinicopathological features of 80 patients with colorectal cancer with high or low expression of DYRK2.

DYRK2 mRNA level

\begin{tabular}{|c|c|c|c|}
\hline Characteristic & Low, n (\%) & High, n (\%) & $\chi^{2}$ P-value \\
\hline \multicolumn{4}{|l|}{ Sex } \\
\hline Male & $28(35.0)$ & $22(27.5)$ & \multirow[t]{2}{*}{0.954} \\
\hline Female & $17(21.2)$ & $13(16.2)$ & \\
\hline \multicolumn{4}{|l|}{ Age, years } \\
\hline$<70$ & $16(20.0)$ & $24(30.0)$ & \multirow[t]{2}{*}{$0.003^{\mathrm{a}}$} \\
\hline$\geq 70$ & $29(36.2)$ & $11(13.7)$ & \\
\hline \multicolumn{4}{|l|}{ Location } \\
\hline Colon & $29(36.2)$ & $14(17.5)$ & \multirow[t]{2}{*}{$0.030^{\mathrm{a}}$} \\
\hline Rectum & $16(20.0)$ & $21(26.2)$ & \\
\hline \multicolumn{4}{|l|}{ Clinical stage } \\
\hline I & $10(12.5)$ & $4(5.0)$ & \multirow[t]{4}{*}{0.240} \\
\hline II & $12(27.5)$ & $13(21.2)$ & \\
\hline III & $16(20.0)$ & $16(20.0)$ & \\
\hline IV & 7 (8.7) & $2(2.5)$ & \\
\hline \multicolumn{4}{|l|}{$\mathrm{T}$ classification } \\
\hline $\mathrm{T} 1+\mathrm{T} 2$ & $11(13.7)$ & $8(10.0)$ & \multirow[t]{2}{*}{0.869} \\
\hline $\mathrm{T} 3+\mathrm{T} 4$ & $34(42.5)$ & $27(33.7)$ & \\
\hline \multicolumn{4}{|l|}{$\mathrm{N}$ classification } \\
\hline No & $23(28.7)$ & $18(22.5)$ & \multirow[t]{2}{*}{0.978} \\
\hline $\mathrm{N} 1+\mathrm{N} 2$ & $22(27.5)$ & $17(21.2)$ & \\
\hline \multicolumn{4}{|c|}{ M classification } \\
\hline M0 & $38(47.5)$ & $33(41.2)$ & \multirow[t]{2}{*}{0.167} \\
\hline M1 & $7(8.7)$ & $2(2.5)$ & \\
\hline \multicolumn{4}{|c|}{ Pathological differentiation } \\
\hline Tub1,2 & $41(51.2)$ & $34(42.5)$ & \multirow[t]{4}{*}{0.486} \\
\hline por & $1(1.2)$ & $1(1.2)$ & \\
\hline pap & $1(1.2)$ & $0(0.0)$ & \\
\hline muc & $2(2.5)$ & $0(0.0)$ & \\
\hline
\end{tabular}

${ }^{a} \mathrm{P}<0.05$. DYRK2, dual specificity tyrosine-phosphorylation-regulated kinase 2; T, tumor; N, node; M, metastasis; tub, tubular adenocarcinoma; por, poorly differentiated adenocarcinoma; muc, mucinous adenocarcinoma; pap, papillary adenocarcinoma.

$\mathbf{A}$

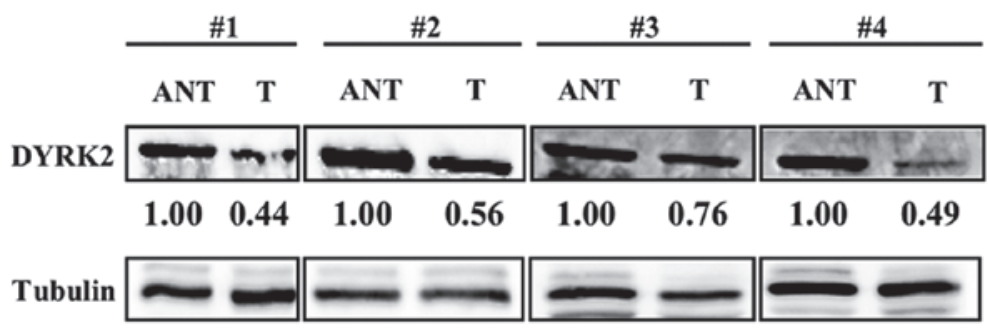

B

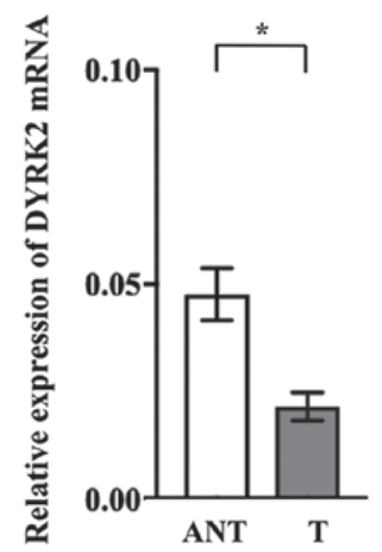

Figure 1. Diminished expression of DYRK2 in colorectal cancer tissues. (A) Western blotting analysis of DYRK2 protein expression in colorectal tumor and ANT ( $\mathrm{n}=4)$. (B) DYRK2 mRNA expression compared with GAPDH in colorectal tumor and ANT using quantitative PCR analysis. $n=80$. "P $<0.05$. T, tumor; ANT, adjacent non-cancerous tissue; DYRK2, dual specificity tyrosine-phosphorylation-regulated kinase 2 . 
$\mathbf{A}$

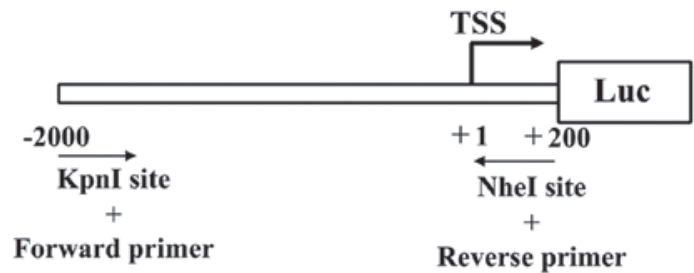

B
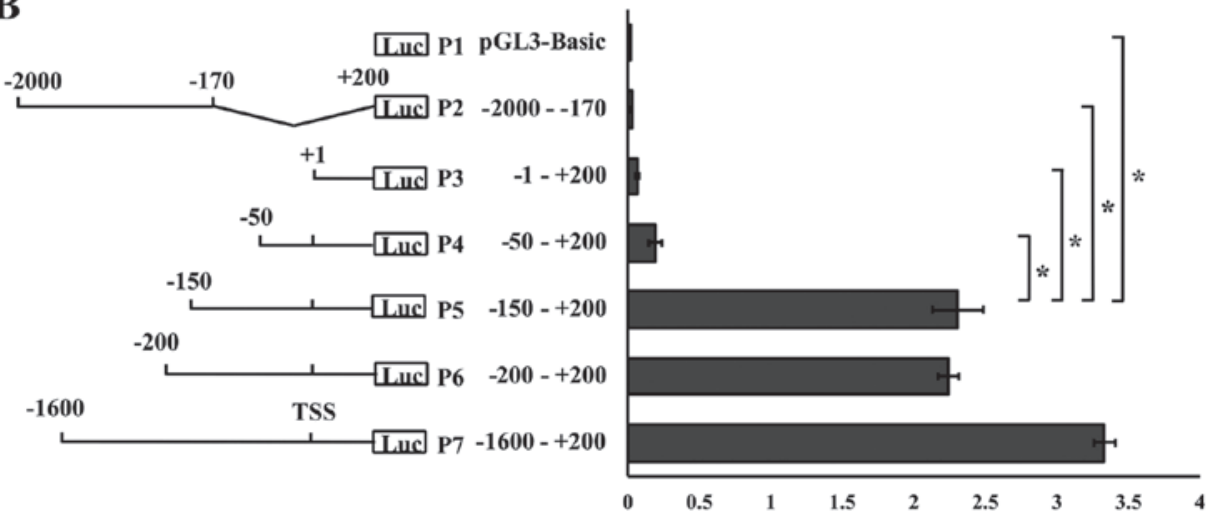

Relative luciferase activity units

C

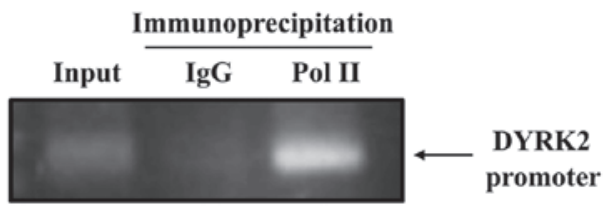

D

$-150$

GGGGACTGAGAGGAGCCGGGCGGGAGGTTGGGTGGGGGTG

GGGTGTGCGTGGCCGCGGCCGGGGGAGCCGGGGGCGGGGC

CTGCGGCGGCGGAGGAGGGCGGCGGAGGGCGGCGGGGGCG

GGGCCGCGCGCCGGCGCCTGTGTGTGAGTGC

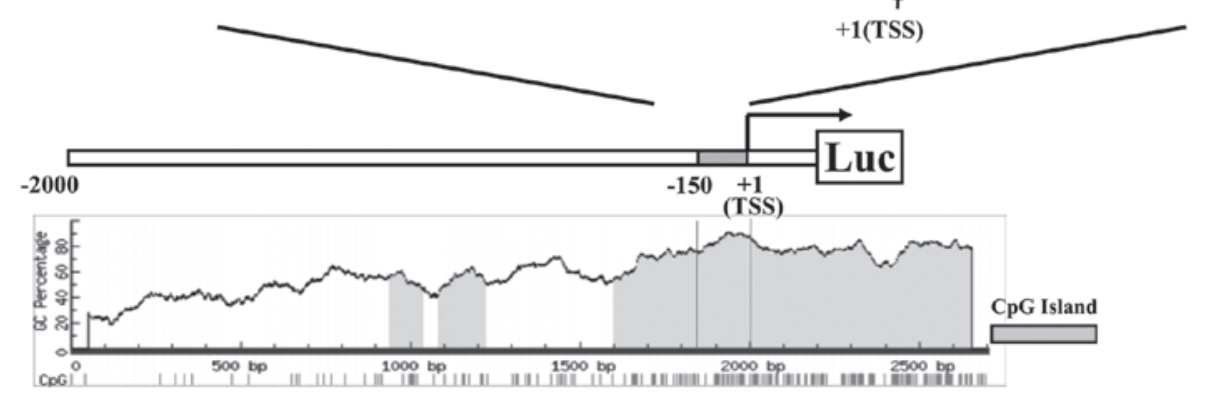

Figure 2. The DYRK2 promoter region is located between 150 bp upstream and 1 bp downstream around the TSS in HCT116 cells. (A) Schematic diagram of the full-length DNA fragment that contains the DYRK2 promoter region. (B) Schematic diagram of various recombinant luciferase reporter constructs (P1-P7, left panel) and relative luciferase reporter activity levels in HCT116 cells (right panel). Data represent results from two independent assays, each performed in triplicate. Reporter activity values are expressed as the mean $\pm \mathrm{SD}$. ${ }^{*} \mathrm{P}<0.01$. (C) To determine whether the region from 150 bp upstream to the TSS is a candidate promoter area of DYRK2, HCT116 cells were stimulated with anti-RNA polymerase II, and a chromatin immunoprecipitation assay with primers of the potential DYRK2 promoter area was performed. Total input DNA and anti-IgG precipitates served as positive and negative controls, respectively. (D) The upper panel represents the DNA sequence of the DYRK2 promoter area. The alignment contained a large number of CpGs. The lower panel demonstrates the DYRK2 promoter area in which the CpG islands are highlighted in gray as predicted by MethPrimer software. DYRK2, dual specificity tyrosine-phosphorylation-regulated kinase 2; TSS, transcription start site; Luc, luciferase.

siRNA (Fig. 3E). The proliferation rates of these cells were analyzed using an MTS assay. Cell proliferation was increased in DYRK2-knockdown HCT116 cells compared with that in cells transfected with a scrambled control (Fig. 3F). By contrast, DYRK2 overexpression suppressed cell proliferation in HCT116 cells (Fig. 3G). To investigate the involvement of DYRK2 promoter methylation in cell proliferation, DYRK2 cells were transfected with siRNA targeting DYRK2 and treated with $5 \mu \mathrm{M}$ Aza. DYRK2-knockdown cells exhibited significantly increased 

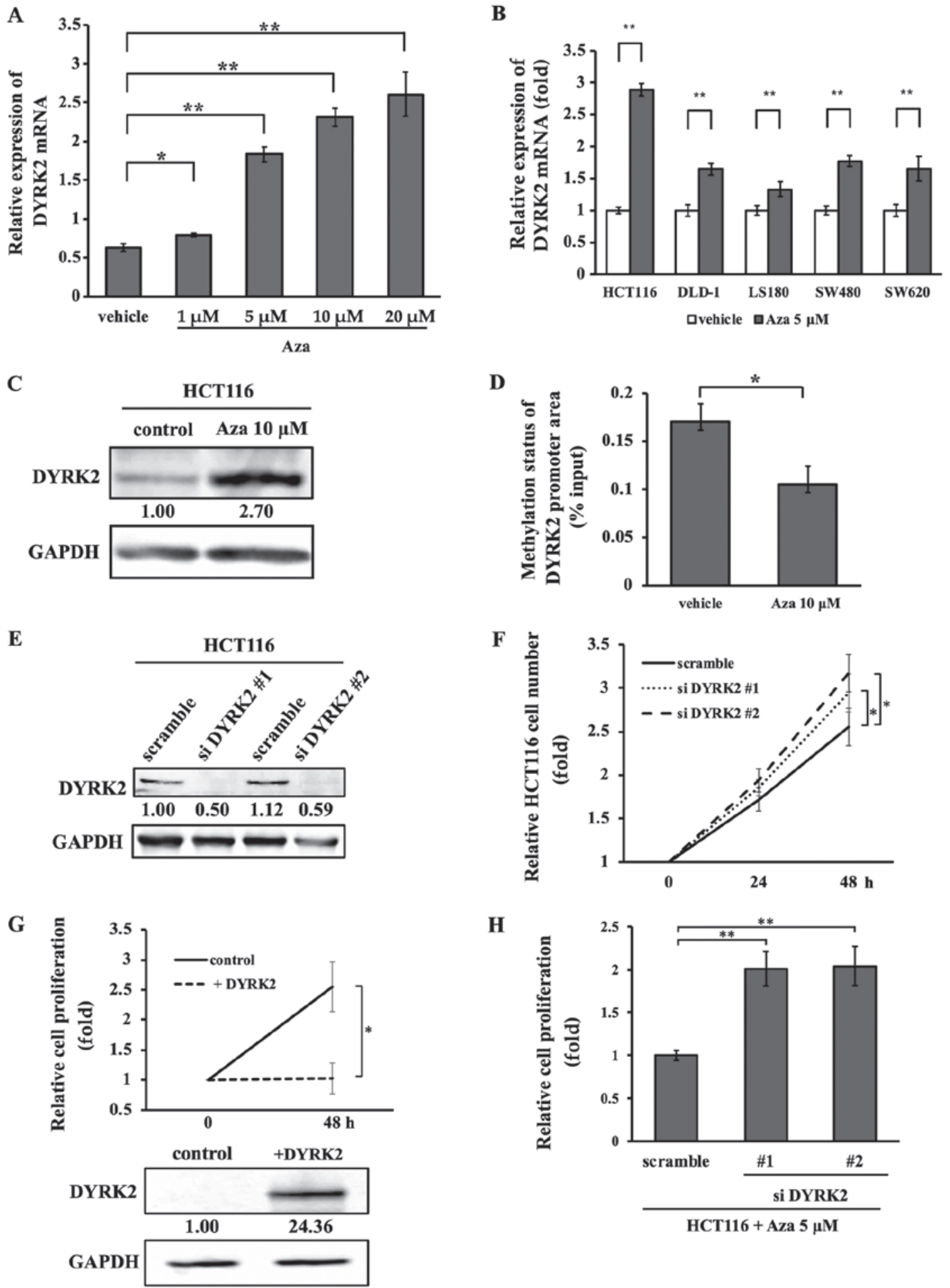

Figure 3. Aza treatment induces the upregulation of DYRK2 expression and results in suppression of cell proliferation. (A) Effects of Aza treatment $(1,5,10$, and $20 \mu \mathrm{M})$ after 1 day on the expression of DYRK2 mRNA in HCT116 cells were determined by quantitative PCR. $\mathrm{n}=3$. (B) The expression of DYRK2 mRNA following $5 \mu$ M Aza treatment in DLD-1, LS180, SW480 and SW620 cells was analyzed by quantitative PCR. n=3. (C) Western blotting demonstrated the alteration of DYRK2 expression 2 days after Aza treatment. (D) Effects of $10 \mu \mathrm{M}$ Aza treatment after 2 days on the methylation status of the DYRK2 promoter area in the HCT116 cells. n=3. (E) HCT116 cells were transfected with two independent DYRK2-specific siRNAs (\#1 and \#2) or a scrambled siRNA. The lysates were analyzed by immunoblotting with anti-DYRK2 or anti-GAPDH antibodies. (F and G) Association between DYRK2 expression and cell proliferation in HCT116 cells. Cell proliferation in (F) transiently DYRK2-depleted or (G) DYRK2-overexpressing cells was measured using an MTS assay. The lysates were analyzed by immunoblotting with anti-DYRK2 or anti-GAPDH antibodies. $n=3$. (H) Cell proliferation was determined in transiently DYRK2-depleted cells compared with the control cells with Aza treatment to confirm the effects of methylation on the DYRK2 promoter area. $\mathrm{n}=3$. Data are presented as the mean $\pm \mathrm{SD} .{ }^{*} \mathrm{P}<0.05,{ }^{* *} \mathrm{P}<0.01$. Aza, 5-Azacytidine; DYRK2, dual specificity tyrosine-phosphorylation-regulated kinase 2 ; siRNA, small interfering RNA; +DYRK2, DYRK2 overexpression vector.

in cell proliferation compared with the control HCT116 cells following Aza treatment (Fig. 3H). These results suggested that the proliferation of HCT116 cells was suppressed by decreased DYRK2 expression with Aza treatment. 


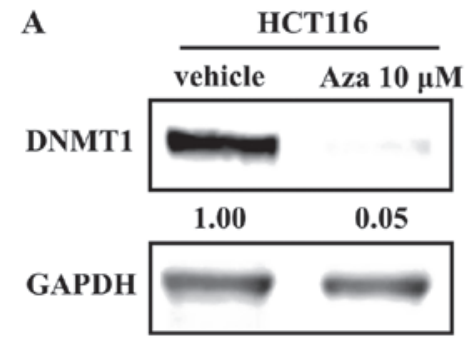

B

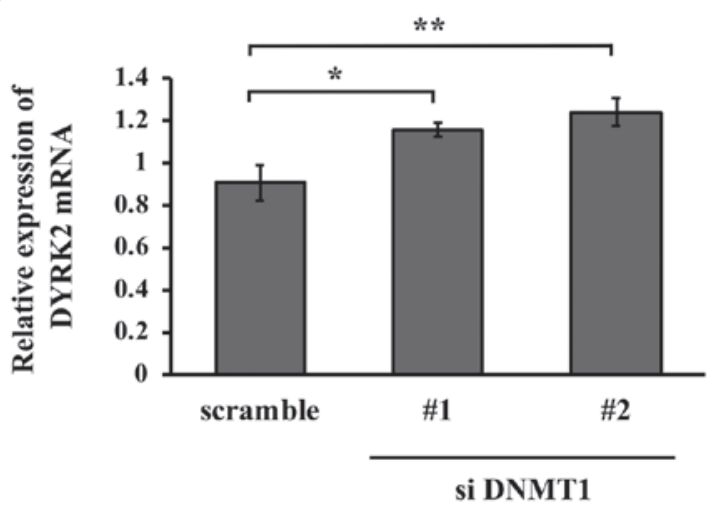

C

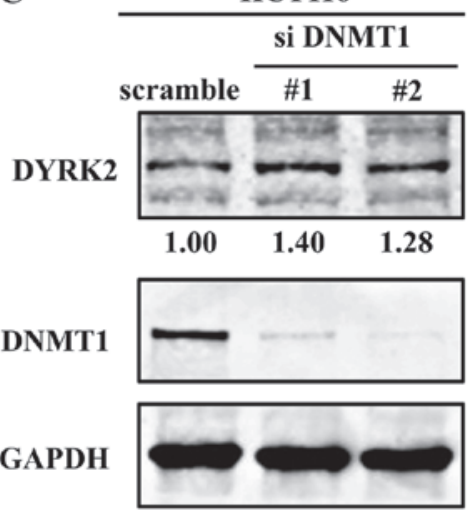

Figure 4. The expression of DYRK2 is regulated by DNMT1 in HCT116 cells. (A) The levels of DNMT1 after $10 \mu$ M Aza treatment were determined by immunoblotting with anti-DNMT1 or anti-GAPDH antibodies. (B) mRNA and (C) protein levels of DYRK2 were assessed in two types of transiently DNMT1-depleted HCT116 cells. GAPDH was used as an internal control. $n=3 .{ }^{*} \mathrm{P}<0.05,{ }^{* * *} \mathrm{P}<0.01$. DYRK2, dual specificity tyrosine-phosphorylation-regulated kinase 2; DNMT1, DNA methyltransferase 1.

Regulation of DYRK2 expression by DNMT1. Aza can lead to DNA hypomethylation by irreversible binding and degradation of DNMT1 (29). The expression of DNMT1was evaluated following treatment with Aza in HCT116 cells to examine the involvement of Aza in DNMT activity. Western blotting results indicated a significant reduction in DNMT1 protein expression by Aza in HCT116 cells (Fig. 4A). Next, the present study determined whether DNMT1 was involved in the regulation of DYRK2 expression at the mRNA and protein levels. Knockdown of DNMT1 by siRNA in HCT116 cells upregulated DYRK2 expression at the mRNA and protein levels (Fig. 4B and C). These results suggested that DYRK2 expression was upregulated by treatment with Aza via the inhibition of DNMT1.

Association between DYRK2 or DNMT1 expression and DYRK2 promoter area methylation status in CRC. An inflammation-associated CRC murine model was used to examine whether the downregulation of DYRK2 expression in $\mathrm{CRC}$ tissue was reproduced in the $\mathrm{CRC}$ model. Therefore, an AOM/DSS model, which is widely applicable to the inflammation-associated colon cancer model in rodents (30-34), was generated. DYRK2 or DNMT1 expression and DYRK2 promoter area methylation status were examined in the AOM/DSS inflammation-associated murine model of CRC. The experimental protocol of AOM/DSS administration is presented in Fig. 5A.

Macroscopic analysis revealed multiple tumors in the colon and rectum, which were frequently observed in the distal colon (Fig. 5B). HE staining demonstrated that the mice had developed colorectal tumors with complicated glandular structures, hyperchromatic nuclei and a loss of nuclear polarity; therefore, these tumors were classified as carcinomas (Fig. 5C). Immunofluorescence analysis of DYRK2 and DNMT1 in colorectal mouse tumors was further performed to investigate the involvement of DYRK2 expression in DNMT1. DYRK2 expression levels were downregulated in the tumor tissue compared with adjacent non-cancerous tissue (Fig. 5C). Of note, focal nuclear staining of DNMT1 was observed in the tumor tissue, whereas an overall cell distribution pattern of DNMT1 was exhibited in the adjacent tissue (Fig. 5C). RT-qPCR of tumor and adjacent non-cancerous tissue was performed to determine the mRNA expression level of DYRK2; consistent with the human samples, DYRK2 mRNA expression was lower in CRC tissue compared with adjacent tissues in the murine model (Fig. 5D).

The degree of methylation at the DYRK2 promoter region was determined in human CRC. A total of 20 pairs of matched CRC and adjacent non-cancerous colorectal tissues, which expressed low levels of DYRK 2 mRNA, were analyzed by quantitative PCR amplification using DNA methylation enrichment methods. The results of the methylation analysis suggested that the degree of methylation at the DYRK2 promoter area was increased in the CRC tissues compared with that in the adjacent non-cancerous tissues (Fig. 5E). These results suggested that DYRK2 expression in CRC was suppressed by DYRK2 promoter region methylation and was involved in the nuclear localization of DNMT1.

\section{Discussion}

The results of the present study demonstrated the molecular mechanism of DYRK2 gene regulation in CRC. These results suggested that the promoter regions of the DYRK2 gene were 
A
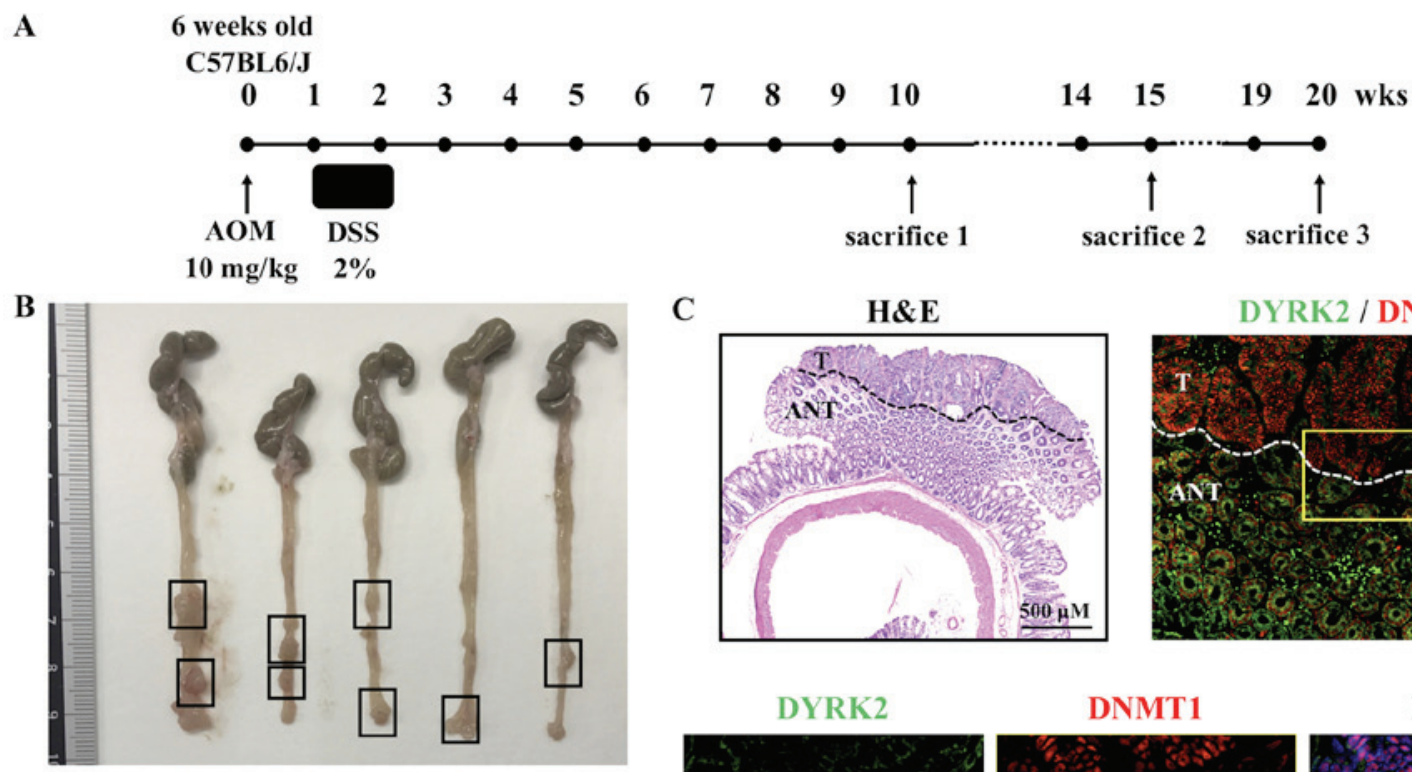

C

H\&E
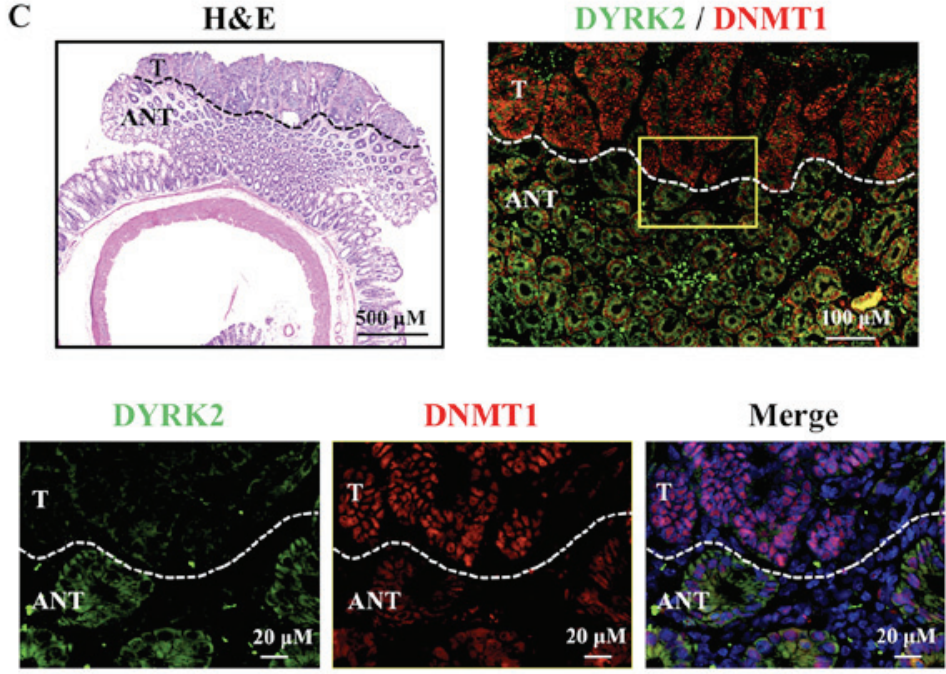

D

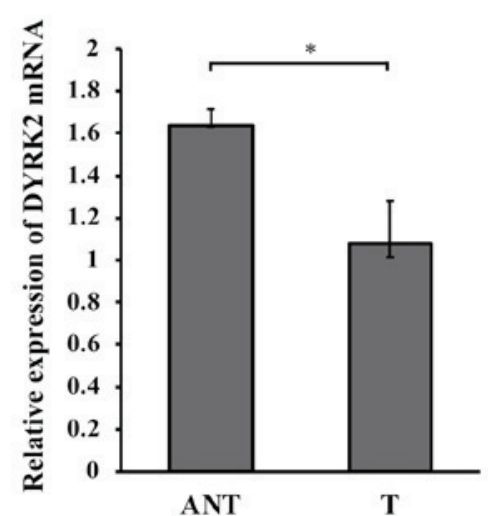

$\mathbf{E}$

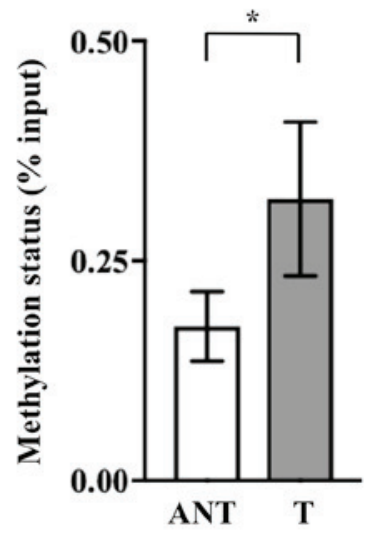

Figure 5. Methylation status of the DYRK2 promoter area is upregulated in CRC tissues. (A) Protocols based on AOM/DSS administration for the induction of colorectal tumor in a murine model. (B) Macroscopic changes in the colorectal tissues. Black arrows indicate colorectal tumors. (C) Representative histopathological (hematoxylin and eosin) and immunofluorescent staining of colorectal tumor and ANT in mice. Confocal microscopy images of immunofluorescent detection of DYRK2 (green) and DNMT1 (red). The nuclei were stained with DAPI (blue). (D) The DYRK2 mRNA expression in colorectal tumors was analyzed and compared with paired ANT from the same mouse by quantitative PCR. $n=3$. ${ }^{*} \mathrm{P}<0.01$. (E) The methylation status of the DYRK2 promoter region in colorectal tumor tissues and ANT determined by DNA methylation analysis. $\mathrm{n}=20$. " $\mathrm{P}<0.05$. ANT, adjacent non-cancerous tissues; DYRK2, dual specificity tyrosine-phosphorylation-regulated kinase 2; DNMT1, DNA methyltransferase 1; CRC, colorectal cancer; AOM, azoxymethane; DSS, dextran sodium sulfate.

methylated in both CRC cell lines and tissues. Suppression of methylation in the DYRK2 promoter region by Aza and knockdown of DNMT1 upregulated DYRK2 expression. Thus, the DYRK2 gene may be a target for aberrant DNA methylation in cancer.

DYRK2 is a tumor suppressive factor that acts via modifications of p53, c-Myc and c-Jun (19,22). DYRK2 knockdown has been demonstrated to promote cell proliferation in breast, ovarian and cervical cancer (35). The present study demonstrated that DYRK2 overexpression led to inhibition of cell proliferation in a CRC cell line. In addition, DYRK2 expression was demonstrated to be regulated at the transcriptional level, as the mRNA level of DYRK2 was significantly decreased in CRC compared with adjacent non-cancerous tissues.
The present study demonstrated that the promoter area of DYRK2 contained a GC-rich genomic sequence and is regulated by DNA methylation. Considering that key tumor suppressor proteins, such as retinoblastoma, p16, von Hippel-Lindau and CDH1, are frequently silenced by DNA methylation within their gene promoters (12), the regulation of DYRK2 by DNA methylation may serve a key role in tumorigenesis.

Aza is a nucleoside inhibitor that was the first hypomethylating agent to be approved by the US Food and Drug Administration for the treatment of myelodysplastic syndrome and acute myeloid leukemia (36). A previous study has reported that Aza disrupts the interaction between DNA and DNMTs (37), and low-dose Aza exerts antitumor effects such as the inhibition of subpopulations of cancer stem-like 
cells (38). The results of the present study demonstrated that Aza treatment decreased the methylation level of DYRK2 promoter area and increased DYRK2 mRNA and protein levels. Furthermore, DYRK2 with increased expression inhibited CRC cell proliferation in vitro. These results suggested the possibility that one of the mechanisms for cell proliferation by Aza treatment was mediated by DNA methylation of DYRK2.

The methylation of mammalian genomic DNA by Aza treatment is catalyzed by DNMTs (29). DNMT1 is the predominant enzyme responsible for the maintenance of DNA methylation (13). In the present study, the mRNA and protein levels of DYRK2 were upregulated by the knockdown of DNMT1 in CRC cells. This result indicated the that DNMT1 may induce the methylation of DYRK2. Overall, the results of the present study suggested that the transcription of DYRK2 was upregulated by treatment with Aza, which induced the inhibition of DNMT1.

A previous study has demonstrated that DNMT1 is localized to the replication foci during the S-phase to duplicate the DNA methylation patterns in daughter strands (39). Further studies have reported that DNMT1 was highly expressed in pancreatic cancer (40), glioblastoma (41), leukemia (42) and colon cancer (43). Of note, the immunofluorescent staining in the present study revealed that DNMT1 was upregulated and primarily localized to the nucleus in mouse CRC tissues. DYRK2 predominantly resided in the cytoplasm in healthy tissue, and the expression was downregulated in the tumor compared with adjacent non-cancerous tissue. These alterations were observed at 10 weeks after AOM/DSS treatment. Therefore, the mechanism of alteration in DNMT localization may be involved in the regulation of DYRK2 expression as well as tumorigenesis and proliferation in CRC. The present study also demonstrated the hypermethylation of the DYRK2 promoter area in human CRC compared with adjacent non-cancerous tissues.

Clinicopathological analysis, including $80 \mathrm{CRC}$ patients, indicated that low mRNA levels of DYRK2 were significantly associated with the tumor location in the colon and patients age $\geq 70$ years. The prognostic prediction of tumor site in CRC remains controversial, and a previous study have indicated that patients with advanced stage rectal cancer exhibited a longer overall survival by 3-4-months compared with those with colon cancer (44). Therefore, the results of the present study suggested that DYRK2 may be a candidate biomarker for prognostic prediction in patients with CRC. However, no significant association was observed between DYRK2 mRNA expression and clinical stage. This may be due to the small number of samples ( 80 patients). Thus, the sample size will be increased in our further studies.

There were some limitations in the current study. First, the exact position of methylation in the DYRK2 promoter region could not be identified. The results demonstrated that the DYRK2 promoter region contained several CpGs, which was determined using a program for PCR primer design. However, the methylation site could not be identified using bisulfite pyrosequencing analysis due to the complex sequence of the DYRK2 promoter region containing a large proportion of $\mathrm{CpG}$ repeat genomic sequences. Therefore, it is necessary to use an alternative approach to identify the methylation position in the DYRK2 promoter. Another limitation is that Aza treatment results in non-specific overall DNA demethylation, which may affect multiple regulatory pathways (29). Therefore, it is necessary to confirm changes in cell proliferation using DYRK2-specific methylation inhibitor in the future.

In conclusion, the results of the present study suggested a regulatory mechanism for cancer cell proliferation by methylation of the DYRK2 promoter area associated with the effects of DNMT1. Identifying new drugs that specifically target DYRK2 and restore DYRK2 expression may be a potential therapeutic strategy for the treatment of CRC.

\section{Acknowledgements}

The authors would like to thank Dr Daisuke Ito (Department of Surgery, The Jikei University School of Medicine) for sampling of the clinical specimens for the in vitro experiments.

\section{Funding}

This study was supported by grants from the Japan Society for the Promotion of Science (KAKENHI; grant nos. 18K15253 and $17 \mathrm{H} 03584$ to K. Yoshida, 18K19484 and 16K18434 to K.Yamada and 19K16781 to S. Yoshida), Japan Agency for Medical Research and Development (grant no. A326TS), the Jikei University Graduate Research Fund for Graduate Students and the Jikei University Research Fund.

\section{Availability of data and materials}

The data sets used and/or analyzed during the current study are available from the corresponding author on reasonable request.

\section{Authors' contributions}

TK and KoY designed and performed the study. SY and SH analyzed the immunofluorescence and immunohistochemistry data. KE made substantial contributions to the analysis and interpretation of the data. KA, KaY and KiY supervised the research. TK, KaY, and KiY wrote the manuscript. All authors read and approved the final manuscript.

\section{Ethics approval and consent to participate}

The study protocol was approved by the Jikei University School of Medicine Ethics Review Committee (approval no. 24-315 7081). All patients provided written informed consent prior to the study.

\section{Patient consent for publication}

Not applicable.

\section{Competing interests}

The authors declare that they have no competing interests.

\section{References}

1. Bray F, Ferlay J, Soerjomataram I, Siegel RL, Torre LA and Jemal A: Global cancer statistics 2018: GLOBOCAN estimates of incidence and mortality worldwide for 36 cancers in 185 countries. CA Cancer J Clin 68: 394-424, 2018. 
2. Fearon ER and Vogelsteine B: A genetic model for colorectal tumorigenesis. Cell 61: 759-767, 1990.

3. Dienstmann R, Vermeulen L, Guinney J, Kopetz S, Tejpar S and Tabernero J: Consensus molecular subtypes and the evolution of precision medicine in colorectal cancer. Nat Rev Cancer 17: 79-92, 2017

4. Goldstein DA, Zeichner SB, Bartnik CM, Neustadter E and Flowers CR: Metastatic colorectal cancer: A systematic review of the value of current therapies. Clin Colorectal Cancer 15: 1-6, 2016.

5. Siegel R, Desantis C and Jemal A: Colorectal cancer statistics, 2014. CA Cancer J Clin 64: 104-117, 2014.

6. Chaffer CL and Weinberg RA: A perspective on cancer cell metastasis. Science 331: 1559-1564, 2011.

7. Manfredi S, Lepage C, Hatem C, Coatmeur O, Faivre J and Bouvier AM: Epidemiology and management of liver metastases from colorectal cancer. Ann Surg 244: 254-259, 2006.

8. Esteller M: Epigenetics in cancer. N Engl J Med 358: 1148-1159, 2008.

9. Simó-Riudalbas L and Esteller M: Cancer genomics identifies disrupted epigenetic genes. Hum Genet 133: 713-725, 2014.

10. Berg M and Søreide K: Genetic and epigenetic traits as biomarkers in colorectal cancer. Int J Mol Sci 12: 9426-9439, 2011.

11. Baylin SB and Jones PA: Epigenetic determinants of cancer. Cold Spring Harb Perspect Biol 8: pii: a019505, 2016.

12. Feinberg AP: The key role of epigenetics in human disease prevention and mitigation. N Engl J Med 378: 1323-1334, 2018.

13. Miremadi A, Oestergaard MZ, Pharoah PD and Caldas C: Cancer genetics of epigenetic genes. Hum Mol Genet 16: R28-R49, 2007.

14. Ferguson-Smith AC and Greally JM: Epigenetics: Perceptive enzymes. Nature 449: 148-149, 2007.

15. Søreide K: Chapter 34 - Cancer Epigenetics. In: Handbook of Epigenetics. 2nd Edition. Academic Press, 2017.

16. Nihira NT and Yoshida K: Engagement of DYRK2 in proper control for cell division. Cell Cycle 14: 802-807, 2015.

17. Taira N, Nihira K, Yamaguchi T, Miki Y and Yoshida K DYRK2 is targeted to the nucleus and controls p53 via Ser46 phosphorylation in the apoptotic response to DNA damage. Mol Cell 25: 725-738, 2007.

18. Yoshida K: Nuclear trafficking of pro-apoptotic kinases in response to DNA damage. Trends Mol Med 14: 305-313, 2008.

19. Taira N, Mimoto R, Kurata M, Yamaguchi T, Kitagawa M, Miki Y and Yoshida K: DYRK2 priming phosphorylation of c-Jun and c-Myc modulates cell cycle progression in human cancer cells. J Clin Invest 122: 859-872, 2012.

20. Maddika S and Chen J: Protein kinase DYRK2 is a scaffold that facilitates assembly of an E3 ligase. Nat Cell Biol 11: 409-419, 2009.

21. Jung HY, Wang X, Jun S and Park JI: Dyrk2-associated EDD-DDB1-VprBP E3 ligase inhibits telomerase by TERT degradation. J Biol Chem 288: 7252-7262, 2013.

22. Mimoto R, Taira N, Takahashi H, Yamaguchi T, Okabe M, Uchida K Miki Y and Yoshida K: DYRK2 controls the epithelial-mesenchymal transition in breast cancer by degrading Snail. Cancer Lett 339: 214-225, 2013.

23. Yamashita S, Chujo M, Moroga T, Anami K, Tokuishi K, Miyawaki M, Kawano Y, Takeno S, Yamamoto S and Kawahara K: DYRK2 expression may be a predictive marker for chemotherapy in non-small cell lung cancer. Anticancer Res 29: 2753-2757, 2009

24. Zhang X, Xu P, Ni W, Fan H, Xu J, Chen Y, Huang W, Lu S, Liang L, Liu J, et al: Downregulated DYRK2 expression is associated with poor prognosis and Oxaliplatin resistance in hepatocellular carcinoma. Pathol Res Pract 212: 162-170, 2016.

25. Yan $\mathrm{H}, \mathrm{Hu} \mathrm{K}, \mathrm{Wu}$ W, Li Y, Tian H, Chu Z, Koeffler HP and Yin D: Low expression of dyrk2 (dual specificity tyrosine phosphorylation regulated Kinase 2) correlates with poor prognosis in colorectal cancer. PLoS One 11: e0159954, 2016.

26. Ito D, Yogosawa S, Mimoto R, Hirooka S, Horiuchi T, Eto K, Yanaga K and Yoshida K: Dual-specificity tyrosine-regulated kinase 2 is a suppressor and potential prognostic marker for liver metastasis of colorectal cancer. Cancer Sci 108: 1565-1573, 2017.
27. Livak KJ and Schmittgen TD: Analysis of relative gene expression data using real-time quantitative PCR and the 2(-Delta Delta C(T)) method. Methods 25: 402-408, 2001.

28. Amin MB, Edge S, Greene F, Byrd DR, Brookland RK, Washington MK, Gershenwald JE, Compton CC, Hess KR, Sullivan DC, et al: AJCC Cancer Staging Manual, 8th edition. Springer, New York, 2017

29. Komashko VM and Farnham PJ: 5-azacytidine treatment reorganizes genomic histone modification patterns. Epigenetics 5: 229-240, 2010

30. Kim M, Miyamoto S, Sugie S, Yasui Y, Ishigamori-Suzuki R, Murakami A, Nakagama $\mathrm{H}$ and Tanaka T: A tobacco-specific carcinogen, NNK, enhances AOM/DSS-induced colon carcinogenesis in male A/J mice. In Vivo 22: 557-563, 2008.

31. Tanaka T, Kohno H, Suzuki R, Yamada Y, Sugie S and Mori H: A novel inflammation-related mouse colon carcinogenesis model induced by azoxymethane and dextran sodium sulfate. Cancer Sci 94: 965-973, 2003

32. Tanaka T, Yasui Y, Tanaka M, Tanaka T, Oyama $\mathrm{T}$ and Rahman KM: Melatonin suppresses AOM/DSS-induced large bowel oncogenesis in rats. Chem Biol Interact 177: 128-136, 2009.

33. Yasui Y, Hosokawa M, Mikami N, Miyashita K and Tanaka T: Dietary astaxanthin inhibits colitis and colitis-associated colon carcinogenesis in mice via modulation of the inflammatory cytokines. Chem Biol Interact 193: 79-87, 2011.

34. Miyoshi N, Nagasawa T, Mabuchi R, Yasui Y, Wakabayashi K, Tanaka $\mathrm{T}$ and Ohshima $\mathrm{H}$ : Chemoprevention of azoxymethane/dextran sodium sulfate-induced mouse colon carcinogenesis by freeze-dried yam sanyaku and its constituent diosgenin. Cancer Prev Res (Phila) 4: 924-934, 2011.

35. Mimoto R, Imawari Y, Hirooka S, Takeyama $H$ and Yoshida $K$ : Impairment of DYRK2 augments stem-like traits by promoting KLF4 expression in breast cancer. Oncogene 36: 1862-1872, 2017.

36. Silverman LR, Demakos EP, Peterson BL, Kornblith AB, Holland JC, Odchimar-Reissig R, Stone RM, Nelson D, Powell BL, DeCastro CM, et al: Randomized controlled trial of azacitidine in patients with the myelodysplastic syndrome: A study of the cancer and leukemia group B. J Clin Oncol 20 : 2429-2440, 2002.

37. Gnyszka A, Jastrzebski Z and Flis S: DNA methyltransferase inhibitors and their emerging role in epigenetic therapy of cancer. Anticancer Res 33: 2989-2996, 2013.

38. Tsai HC, Li H, Van Neste L, Cai Y, Robert C, Rassool FV, Shin JJ, Harbom KM, Beaty R, Pappou E, et al: Transient low doses of DNA-demethylating agents exert durable antitumor effects on hematological and epithelial tumor cells. Cancer Cell 21: 430-446, 2012.

39. Probst AV, Dunleavy E and Almouzni G: Epigenetic inheritance during the cell cycle. Nat Rev Mol Cell Biol 10: 192-206, 2009.

40. He S, Wang F, Yang L, Guo C, Wan R, Ke A, Xu L, Hu G, Xu X Shen J and Wang X: Expression of DNMT1 and DNMT3a are regulated by GLI1 in human pancreatic cancer. PLoS One 6: e27684, 2011

41. Rajendran G, Shanmuganandam K, Bendre A, Muzumdar D, Goel A and Shiras A: Epigenetic regulation of DNA methyltransferases: DNMT1 and DNMT3B in gliomas. J Neurooncol 104: 483-494, 2011

42. Mizuno S, Chijiwa T, Okamura T, Akashi K, Fukumaki Y, Niho Y and Sasaki H: Expression of DNA methyltransferases DNMT1, 3A, and 3B in normal hematopoiesis and in acute and chronic myelogenous leukemia. Blood 97: 1172-1179, 2001.

43. Robertson KD, Uzvolgyi E, Liang G, Talmadge C, Sumegi J, Gonzales FA and Jones PA: The human DNA methyltransferases (DNMTs) 1, 3a and 3b: Coordinate mRNA expression in normal tissues and overexpression in tumors. Nucleic Acids Res 27: 2291-2298, 1999.

44. Lee YC, Lee YL, Chuang JP and Lee JC: Differences in survival between colon and rectal cancer from SEER data. PLoS One 8: e78709, 2013. 